PROCEEDINGS OF THE

AMERICAN MATHEMATICAL SOCIETY

Volume 138, Number 5, May 2010, Pages 1587-1593

S 0002-9939(10)10201-9

Article electronically published on January 6, 2010

\title{
AUSLANDER GENERATORS OF ITERATED TILTED ALGEBRAS
}

\author{
FLÁVIO ULHOA COELHO, DIETER HAPPEL, AND LUISE UNGER
}

(Communicated by Birge Huisgen-Zimmermann)

\begin{abstract}
Let $\Lambda$ be an iterated tilted algebra. We will construct an Auslander generator $M$ in order to show that the representation dimension of $\Lambda$ is three in case $\Lambda$ is representation infinite.
\end{abstract}

Recently there has been a lot of attention to compute the representation dimension of a finite dimensional algebra $\Lambda$, a notion introduced by Auslander in $\mathrm{A}$ ] in an attempt to measure the complexity of the representation theory of $\Lambda$. It seems from the results obtained in the last few years that it actually measures the homological complexity of $\Lambda$, and we want to provide some further evidence.

We will not need the original definition, but rather the following characterization already going back to Auslander in [A]; see also [EHIS] or [CP] for a more detailed account.

For this let $\Lambda$ be an arbitrary finite dimensional algebra. Let $\bmod \Lambda$ be the category of finitely generated left $\Lambda$-modules. Let $M \in \bmod \Lambda$ be a generatorcogenerator. So we have that ${ }_{\Lambda} \Lambda \oplus D \Lambda_{\Lambda} \in$ add $M$, where $D$ is the standard duality on $\bmod \Lambda$ and add $M$ is the full subcategory of $\bmod \Lambda$ containing the direct summands of direct sums of $M$. Let $d$ be the minimum such that there is a generatorcogenerator with the following property: For each $X \in \bmod \Lambda$ there is an exact sequence

$$
\begin{gathered}
0 \rightarrow M^{d} \rightarrow \cdots \rightarrow M^{1} \rightarrow M^{0} \rightarrow X \rightarrow 0 \text { such that } \\
0 \rightarrow \operatorname{Hom}_{\Lambda}\left(M, M^{d}\right) \rightarrow \cdots \rightarrow \operatorname{Hom}_{\Lambda}\left(M, M^{0}\right) \rightarrow \operatorname{Hom}_{\Lambda}(M, X) \rightarrow 0
\end{gathered}
$$

is exact, where $M^{i} \in$ add $M$ for $0 \leq i \leq t$. Then the representation dimension rep.dim $\Lambda$ of $\Lambda$ is $d+2$. It follows from Iyama's result [I] that the representation dimension of $\Lambda$ is always finite. Trivially, if $\Lambda$ is representation finite, then the representation dimension of $\Lambda$ is two. We call a generator-cogenerator where the minimum is attained an Auslander generator.

As the main result of this article we will show here that for an iterated tilted algebra $\Lambda$ there is a generator-cogenerator $M$ such that $d=1$. We will recall the definition of this class of algebras below. In particular we obtain that an iterated

Received by the editors April 7, 2009, and, in revised form, July 24, 2009.

2000 Mathematics Subject Classification. Primary 16E05, 16E10, 16G10.

The results presented here were obtained while the second and third authors were visiting IME-USP. They thank their coauthor for his kind hospitality during their pleasant stay in São Paulo. The project was made possible by a grant from FAPESP, Brazil. The first author also acknowledges a grant from $\mathrm{CNP}_{q}$. 
tilted algebra which is representation infinite has representation dimension three. Note that this generalizes the result for tilted algebras in APT. For related results we refer to [O] and the literature quoted there. We will include an easy example of an iterated tilted algebra $\Lambda$ showing that no proper direct summand $M^{\prime}$ of $M$ which still is a generator-cogenerator will have this property.

We now recall the concept of iterated tilted algebras. For this let $H$ be a finite dimensional hereditary $k$-algebra over a field $k$. Let $\bmod H$ be the category of finitely generated left $H$-modules. Iterated tilted algebras were introduced in $\mathrm{AH}$ under the name of generalized tilted algebras. We will not use the original definition, but instead will use the characterization given in HRS. For this consider the bounded derived category $D^{b}(H)$ of $\bmod H$. Then the result in [HRS states that a finite dimensional $k$-algebra is an iterated tilted algebra if and only if the derived category $D^{b}(\Lambda)$ of the category $\bmod \Lambda$ of finitely generated left $\Lambda$-modules is equivalent as a triangulated category to $D^{b}(H)$ for some finite dimensional hereditary $k$-algebra $H$, or equivalently, using [Ri], that there is a tilting complex $T^{\bullet} \in D^{b}(H)$ such that $\Lambda \simeq \operatorname{End}_{D^{b}(H)} T^{\bullet}$. We call $H$ the type of $\Lambda$, which is only defined up to derived equivalence. So the class of iterated tilted algebras coincides with the class of piecewise hereditary algebras of type $H$. In general the class of piecewise hereditary algebras is defined to be those algebras being derived equivalent to hereditary, abelian categories. It follows from [H2] that there is only one other class of piecewise hereditary algebras, namely those of type $\operatorname{coh} \mathbb{X}$ for a weighted projective line in the sense of GL2. Our result should also be true there, but the absence of indecomposable projectives and indecomposable injectives in coh $\mathbb{X}$ shows that our methods used in the second section will fail.

In the first section we will briefly recall some useful facts on iterated tilted algebras. In section two we then will present the construction of a generatorcogenerator with the property mentioned above and show the main result.

We denote the composition of morphisms $f: X \rightarrow Y$ and $g: Y \rightarrow Z$ in a given category $\mathcal{K}$ by $f g$. The notation and terminology introduced here will be fixed throughout this article. For unexplained representation-theoretic and derived category terminology, we refer to [ARS, [H1] and [R].

\section{Preliminaries}

In this section we briefly recall some useful facts on iterated tilted algebras which will be used in the next section. We keep the notation from the introduction. Let $T^{\bullet} \in D^{b}(H)$ be a tilting complex and let $\Lambda=\operatorname{End}_{D^{b}(H)} T^{\bullet}$. If $X \in D^{b}(H)$ is indecomposable, then it is well known that $X \in(\bmod H)[i]$ for some $i \in \mathbb{Z}$, where we denote by [.] the shift functor on $D^{b}(H)$ and identify $\bmod H$ with $(\bmod H)[0]$, the stalk complexes concentrated in degree zero. Then up to shift we may assume that $T^{\bullet}=\bigoplus_{t=0}^{r} T_{t}[t]$, where $T_{t} \in \bmod H$. We denote by $F: D^{b}(H) \rightarrow D^{b}(\Lambda)$ the triangle equivalence induced by $T^{\bullet}$ and by $G$ a quasi inverse to $F$. Thus for any indecomposable $\Lambda$-module $X$ we have that $G(X) \in \bigcup_{t=0}^{r+1}(\bmod H)[t]$. For $0 \leq t \leq$ $r+1$ we denote by $\mathcal{U}_{t}$ the full subcategory of $\bmod \Lambda$ with objects the direct sums of those indecomposable $\Lambda$-modules $X$ such that $G(X) \in(\bmod H)[t]$. We refer to [H1] for properties and more details of the subcategories $\mathcal{U}_{t}$. In particular we will use that $\operatorname{Hom}_{\Lambda}(X, Y)=0$ for $X \in \mathcal{U}_{s}, Y \in \mathcal{U}_{t}$ such that $|s-t| \geq 2$.

We will also need the following notation. For $X \in \bmod H$ we denote by $\mathcal{T}(X)$ the full subcategory of $\bmod H$ containing those $Y$ such that $\operatorname{Ext}_{H}^{1}(X, Y)=0$ and by 
$\mathcal{F}(X)$ the full subcategory of $\bmod H$ containing those $Y$ such that $\operatorname{Hom}_{H}(X, Y)=0$. So the right orthogonal category as defined in GL1] is $X^{\text {perp }}=\mathcal{T}(X) \cap \mathcal{F}(X)$.

The next lemma collects some basic information on iterated tilted algebras; for details, see [S].

Lemma 1.1. Let $\Lambda$ be an iterated tilted algebra given by a tilting complex $T^{\bullet}$ and a triangle equivalence $F$ as above. Then

(i) For $i \neq j$ we have that $\operatorname{Hom}_{H}\left(T_{i}, T_{j}\right)=0$ and for $j \neq i+1$ we have that $\operatorname{Ext}_{H}^{1}\left(T_{i}, T_{j}\right)=0$.

(ii) For $0 \leq t \leq r+1$, let $\mathcal{V}_{t} \subset \bmod H$ be defined as

$$
\mathcal{V}_{t}=\bigcap_{i \neq t, t-1} T_{i}^{\text {perp }} \cap \mathcal{T}\left(T_{t}\right) \cap \mathcal{F}\left(T_{t-1}\right) .
$$

Then the restriction of $F$ to $\mathcal{V}_{t}[t]$ induces an equivalence of $\mathcal{V}_{t}[t]$ with $\mathcal{U}_{t}$.

In the next proposition we collect some further properties of the subcategories $\mathcal{V}_{t} \subset \bmod H$ as defined in Lemma 1.1 The first two assertions follow easily from Lemma 1.1(ii), and the last is contained in [HZ]. For the notion of functorial finiteness of a given subcategory of $\bmod \Lambda$ we refer to [AS2].

Proposition 1.2. Let $\Lambda$ be an iterated tilted algebra given by a tilting complex $T^{\bullet}$ and a triangle equivalence $F$ as above.

(i) For $0 \leq t \leq r+1$, the subcategories $\mathcal{V}_{t}$ are closed under extensions, direct summands, direct sums and images.

(ii) Let $0 \rightarrow X \rightarrow Y \rightarrow Z \rightarrow 0$ be an exact sequence in $\bmod H$ with $Y, Z \in \mathcal{V}_{t}$ and $\operatorname{Ext}_{H}^{1}\left(T_{t}, X\right)=0$; then $X \in \mathcal{V}_{t}$.

(iii) For $0 \leq t \leq r+1$, the subcategories $\mathcal{V}_{t}$ are functorially finite in mod $H$.

\section{Construction of an Auslander generator}

We keep the notation from the previous section. Fix an integer $0 \leq t \leq r+1$. We consider the subcategory $\mathcal{V}_{t} \subset \bmod H$. By Proposition 1.2 we know that $\mathcal{V}_{t}$ is functorially finite in $\bmod H$. Let ${ }_{H} H \rightarrow G_{t}$ be a minimal left $\mathcal{V}_{t}$-approximation of ${ }_{H} H$ and let $E_{t} \rightarrow D H_{H}$ be a minimal right $\mathcal{V}_{t}$-approximation of $D H_{H}$. By AS2] we know that $G_{t}$ is Ext-projective in $\mathcal{V}_{t}$, so $\operatorname{Ext}_{H}^{1}\left(G_{t}, X\right)=0$ for all $X \in \mathcal{V}_{t}$, and that $E_{t}$ is Ext-injective in $\mathcal{V}_{t}$, so $\operatorname{Ext}_{H}^{1}\left(X, E_{t}\right)=0$ for all $X \in \mathcal{V}_{t}$. Let $n$ be the number of distinct simple $H$-modules. Since $H$ is hereditary we know that an Ext-injective in $\mathcal{V}_{t}$ is a partial tilting module, so has at most $n$ indecomposable direct summands up to isomorphism. Thus we may consider $F_{t}$ the direct sum of all indecomposable Ext-injectives in $\mathcal{V}_{t}$ up to isomorphism. Similarly we can define $R_{t}$ as the direct sum of all indecomposable Ext-projectives in $\mathcal{V}_{t}$ up to isomorphism. It follows from Lemma 1.1 that $T_{t} \in$ add $R_{t}$.

Let $T^{\bullet} \in D^{b}(H)$ be the tilting complex and let $\Lambda=\operatorname{End}_{D^{b}(H)} T^{\bullet}$. Moreover let $F: D^{b}(H) \rightarrow D^{b}(\Lambda)$ be the triangle equivalence induced by $T^{\bullet}$. For $0 \leq t \leq r+1$ set $Q_{t}=F\left(R_{t}[t]\right)$ and $L_{t}=F\left(F_{t}[t]\right)$. Finally, let $M=\bigoplus_{t=0}^{r+1} M_{t}$, where $M_{t}=Q_{t} \oplus L_{t}$. Clearly, if $X_{t}$ is Ext-projective in $\mathcal{U}_{t}$, then $X_{t} \in$ add $Q_{t}$ and, if $Y_{t}$ is Ext-injective in $\mathcal{U}_{t}$, then $Y_{t} \in \operatorname{add} L_{t}$. So ${ }_{\Lambda} \Lambda \in$ add $\bigoplus_{t=0}^{r+1} Q_{t}$ and $D \Lambda_{\Lambda} \in$ add $\bigoplus_{t=0}^{r+1} L_{t}$; hence the following is immediate.

Lemma 2.1. The module $M$ is a generator-cogenerator for $\bmod \Lambda$. 
Note that our choice of $M$ does not only depend on $\Lambda$, but also on the concrete realization of $\Lambda$ as an endomorphism algebra of a tilting complex in $D^{b}(H)$.

We will now show certain useful properties of the $H$-modules $R_{t}$ and $F_{t}$.

Proposition 2.2. Let $0 \leq t \leq r+1$ and $X \in \mathcal{V}_{t}$.

(i) Let $\pi: R_{t}^{0} \rightarrow X$ be a minimal right add $R_{t}$-approximation of $X$. Then $\pi$ is surjective and $k e r \pi \in$ add $R_{t}$.

(ii) Let $\alpha: \tilde{F}_{t} \rightarrow X$ be a minimal right add $F_{t}$-approximation of $X$. Then $\alpha$ is injective.

Proof. First we show (i). Let $X \in \mathcal{V}_{t}$. Let $\rho: P(X) \rightarrow X$ be the projective cover of $X$ and $h: P(X) \rightarrow \tilde{R}_{t}$ be a minimal left $\mathcal{V}_{t}$-approximation of $P(X)$. Then $\tilde{R}_{t} \in$ add $R_{t}$ and so there is $g: \tilde{R}_{t} \rightarrow X$ such that $\rho=h g$. Since $\rho$ is surjective, we infer that $g$ is surjective. So a minimal right add $R_{t}$-approximation $\pi: R_{t}^{0} \rightarrow X$ of $X$ is surjective. Since $T_{t} \in \operatorname{add} R_{t}$, we have that $\operatorname{Hom}\left(T_{t}, \pi\right)$ is surjective, and since $\operatorname{Ext}_{H}^{1}\left(T_{t}, R_{t}\right)=0$ we have that $\operatorname{Ext}_{H}^{1}\left(T_{t}, \operatorname{ker} \pi\right)=0$. So by Proposition 1.2(ii) we infer that $\operatorname{ker} \pi \in \mathcal{V}_{t}$. Since $H$ is hereditary we see that $\operatorname{Ext}_{H}^{1}\left(R_{t}^{0}, Y\right) \rightarrow \operatorname{Ext}_{H}^{1}(\operatorname{ker} \pi, Y)$ is surjective, so $\operatorname{Ext}_{H}^{1}(\operatorname{ker} \pi, Y)=0$ for $Y \in \mathcal{V}_{t}$, so ker $\pi \in$ add $R_{t}$.

For (ii) consider a minimal right add $F_{t}$-approximation $\alpha: \tilde{F}_{t} \rightarrow X$ of $X$. If $\alpha$ is not injective, we consider $\alpha=\rho \mu$, where $\rho: \tilde{F}_{t} \rightarrow \operatorname{im} \alpha$ is a proper surjection and $\mu$ is the inclusion of $\operatorname{im} \alpha \rightarrow X$. By Proposition 1.2(i) we have that $\operatorname{im} \alpha \in \mathcal{V}_{t}$. Since $H$ is hereditary we have that $\operatorname{Ext}_{H}^{1}\left(Y, \tilde{F}_{t}\right) \rightarrow \operatorname{Ext}_{H}^{1}(Y, \operatorname{im} \alpha)$ is surjective, so $\operatorname{Ext}_{H}^{1}(Y, \operatorname{im} \alpha)=0$ for $Y \in \mathcal{V}_{t}$, so im $\alpha \in \operatorname{add} F_{t}$. Since $\operatorname{Hom}_{H}\left(F_{t}, \tilde{F}_{t}\right) \rightarrow$ $\operatorname{Hom}_{H}\left(F_{t}, X\right)$ is surjective we infer that also $\operatorname{Hom}_{H}\left(F_{t}, \operatorname{im} \alpha\right) \rightarrow \operatorname{Hom}_{H}\left(F_{t}, X\right)$ is surjective. So $\mu$ is a right add $F_{t}-$ approximation of $X$ of smaller length, which contradicts the minimality of $\alpha$; hence $\alpha$ is injective.

Corollary 2.3. Let $0 \leq t \leq r+1$ and let $X \in \mathcal{V}_{t}$. Then there is a short exact sequence

$$
0 \longrightarrow R_{t}^{1} \longrightarrow N_{t} \stackrel{f}{\longrightarrow} X \longrightarrow 0
$$

with $f$ a right add $\left(R_{t} \oplus F_{t}\right)$-approximation of $X$ and $R_{t}^{1} \in$ add $R_{t}$.

Proof. Let $X \in \mathcal{V}_{t}$. From Proposition 2.2(i) we obtain a short exact sequence

$$
0 \longrightarrow R_{t}^{1} \longrightarrow R_{t}^{0} \stackrel{\pi}{\longrightarrow} X \longrightarrow 0
$$

with $R_{t}^{0}, R_{t}^{1} \in$ add $R_{t}$ and $\pi$ a minimal right add $R_{t}-$ approximation of $X$. By Proposition 2.2(ii) we have that a minimal right add $F_{t}$-approximation $\alpha: \tilde{F}_{t} \rightarrow X$ is injective. So we consider the following pullback diagram:

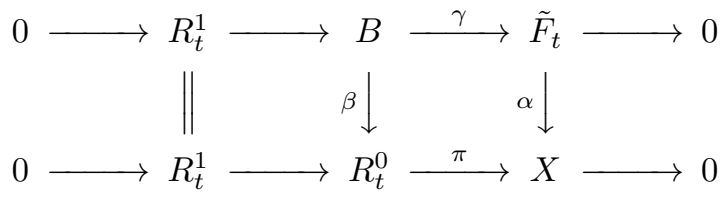

Since $\alpha$ is injective, we see that $\beta$ is injective. Since $\mathcal{V}_{t}$ is closed under extensions, we infer that $B \in \mathcal{V}_{t}$. Since $H$ is hereditary we see that $\operatorname{Ext}_{H}^{1}\left(R_{t}^{0}, Y\right) \rightarrow \operatorname{Ext}_{H}^{1}(B, Y)$ is surjective, so that $\operatorname{Ext}_{H}^{1}(B, Y)=0$ for $Y \in \mathcal{V}_{t}$, so $B \in$ add $R_{t}$. Clearly,

$$
f=\left(\begin{array}{l}
\alpha \\
\pi
\end{array}\right): \tilde{F}_{t} \oplus R_{t}^{0} \rightarrow X
$$


is a right add $\left(F_{t} \oplus R_{t}\right)$-approximation of $X$. Since $(*)$ is a pullback diagram we obtain a short exact sequence

$$
0 \longrightarrow B \longrightarrow \tilde{F}_{t} \oplus R_{t}^{0} \stackrel{f}{\longrightarrow} X \longrightarrow 0
$$

with $f$ a right add $\left(R_{t} \oplus F_{t}\right)$-approximation of $X$ and $B \in$ add $R_{t}$, thus showing the assertion.

Lemma 2.4. Let $\Lambda$ be an iterated tilted algebra with pieces $\mathcal{U}_{0}, \ldots, \mathcal{U}_{r+1}$. For $0 \leq$ $t \leq r+1$, let $Q_{t}, L_{t} \in \mathcal{U}_{t}$ be as above. Let $X \in \mathcal{U}_{t}$ and $Y \in \mathcal{U}_{t+1}$ for $0 \leq t \leq r$.

(i) Any map $f: X \rightarrow Y$ factors over add $Q_{t+1}$.

(ii) Any map $f: X \rightarrow Y$ factors over add $L_{t}$.

Proof. We show the first assertion. The second assertion follows by dual arguments. So let $X \in \mathcal{U}_{t}$ and $Y \in \mathcal{U}_{t+1}$ for $0 \leq t \leq r$ and let $f: X \rightarrow Y$ be a nonzero map. Let $X^{\prime}[t]=G(X)$ and $Y^{\prime}[t+1]=G(Y)$ for $X^{\prime} \in \mathcal{V}_{t}$ and $Y^{\prime} \in \mathcal{V}_{t+1}$. Then

$$
G(f) \in \operatorname{Hom}_{D^{b}(H)}\left(X^{\prime}[t], Y^{\prime}[t+1]\right) \simeq \operatorname{Hom}_{D^{b}(H)}\left(X^{\prime}, Y^{\prime}[1]\right) .
$$

Let $\pi: P\left(Y^{\prime}\right) \rightarrow Y^{\prime}$ be the projective cover of $Y^{\prime}$. Since $H$ is hereditary we have that $\operatorname{Ext}^{1}\left(X^{\prime}, \pi\right): \operatorname{Ext}^{1}\left(X^{\prime}, P\left(Y^{\prime}\right)\right) \rightarrow \operatorname{Ext}^{1}\left(X^{\prime}, Y^{\prime}\right)$ is surjective. Since $\operatorname{Ext}_{H}^{1}\left(X^{\prime}, Y^{\prime}\right) \simeq \operatorname{Hom}_{D^{b}(H)}\left(X^{\prime}, Y^{\prime}[1]\right)$, we see that there is $h: X^{\prime}[t] \rightarrow P\left(Y^{\prime}\right)[t+1]$ such that $h \pi[t+1]=G(f)$. Let $g: P\left(Y^{\prime}\right) \rightarrow \tilde{R}_{t+1}$ be a minimal left $\mathcal{V}_{t+1}-$ approximation of $P\left(Y^{\prime}\right)$. Then $\tilde{R}_{t+1} \in$ add $R_{t+1}$. Thus there is $h^{\prime}: \tilde{R}_{t+1} \rightarrow Y^{\prime}$ such that $g h^{\prime}=\pi$. So $G(f)=h \pi[t+1]=h g[t+1] h^{\prime}[t+1]$. So using the equivalence $F$ we see that $f=F G(f)=F(h g[t+1]) F\left(h^{\prime}[t+1]\right)$ and $F\left(\tilde{R}_{t+1}\right) \in$ add $Q_{t+1}$, so $f$ factors over add $Q_{t+1}$.

Theorem 2.5. Let $\Lambda$ be an iterated tilted algebra and let $M=\bigoplus_{t=0}^{r+1} M_{t}$, where $M_{t}=\left(Q_{t} \oplus L_{t}\right)$ for $0 \leq t \leq r+1$. Then $M$ is a generator-cogenerator and for each $X \in \bmod \Lambda$ there exists an exact sequence $0 \rightarrow M^{1} \rightarrow M^{0} \rightarrow X \rightarrow 0$ with $M^{0}, M^{1} \in$ add $M$ such that $0 \rightarrow \operatorname{Hom}_{\Lambda}\left(M, M^{1}\right) \rightarrow \operatorname{Hom}_{\Lambda}\left(M, M^{0}\right) \rightarrow \operatorname{Hom}_{\Lambda}(M, X) \rightarrow 0$ is exact.

Proof. By Lemma 2.1 we know that $M$ is a generator-cogenerator. It is clearly enough to construct such a sequence for a $\Lambda$-module $X \in \mathcal{U}_{t}$ for $0 \leq t \leq r+1$. Since $X \in \mathcal{U}_{t}$ there is $Y \in \mathcal{V}_{t}$ such that $X=F(Y[t])$. Using Corollary 2.3 we obtain a short exact sequence

$$
0 \longrightarrow R_{t}^{1} \longrightarrow N_{t} \stackrel{f}{\longrightarrow} Y \longrightarrow 0
$$

with $f$ a right add $\left(R_{t} \oplus F_{t}\right)$-approximation of $Y$ and $R_{t}^{1} \in$ add $R_{t}$. Then we obtain an exact sequence of $\Lambda$-modules

$$
0 \longrightarrow F\left(R_{t}^{1}[t]\right) \longrightarrow F\left(N_{t}[t]\right) \stackrel{F(f[t])}{\longrightarrow} F(Y[t]) \longrightarrow 0
$$

with $g=F(f[t])$ a right add $\left(Q_{t} \oplus L_{t}\right)$-approximation of $X$ and also $F\left(R_{t}^{1}[t]\right) \in$ add $Q_{t}$. Now $\operatorname{Hom}_{\Lambda}\left(M_{i}, X\right)=0$ for $i>t$ and $i<t-1$. By Lemma 2.4 any map from $M_{t-1}$ to $X$ factors over add $Q_{t}$, so we infer that $g$ is an add $M$-approximation of $X$. But then $\operatorname{Hom}_{\Lambda}(M, g)$ is surjective, which shows the assertion.

Example 2.6. Next we give an example of an iterated tilted algebra $\Lambda$ showing that no proper direct summand $M^{\prime}$ of $M$ which still is a generator-cogenerator will 
have this property. For this consider $H$ to be the path algebra over $k$ of the linearly oriented quiver $\mathbb{A}_{6}$,

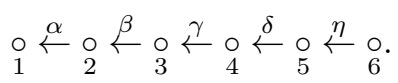

We choose $T_{0}=S(2), T_{1}=S(1) \oplus P(3) \oplus P(4) \oplus P(6) \oplus S(6)$, where $S(i)$ is the simple $H$-module associated with the vertex $i$ and $P(i)$ its projective cover. Then $T^{\bullet}=T_{0}[0] \oplus T_{1}[1] \in D^{b}(H)$ is a tilting complex with $\Lambda=\operatorname{End}_{D^{b}(H)} T^{\bullet}$ the quotient of $H$ by the two-sided ideal generated by $\beta \alpha$ and $\eta \delta$. Now $\mathcal{U}_{0}=$ add $S(1)$ and $\mathcal{U}_{2}=$ add $S(6)$, while all other indecomposable $\Lambda$-modules belong to $\mathcal{U}_{1}$. Our choice of $M$ in this case is $M={ }_{\Lambda} \Lambda \oplus D \Lambda_{\Lambda} \oplus S(2) \oplus S(5)$. It is straightforward to check that no proper direct summand $M^{\prime}$ of $M$ which is still a generator-cogenerator will satisfy the assertion of Theorem 2.5 .

As mentioned in the introduction the following is an immediate consequence of Theorem 2.5.

Corollary 2.7. Let $\Lambda$ be an iterated tilted algebra which is representation infinite. Then the representation dimension of $\Lambda$ is three.

It is well known that an iterated tilted algebra of type $H$, where $H$ is a representation finite hereditary algebra is again representation finite. So also the following is an immediate consequence of Theorem 2.5. A direct proof of this could provide some further insight.

Corollary 2.8. Let $\Lambda$ be an iterated tilted algebra of type $H$. Then rep.dim $\Lambda \leq$ rep. $\operatorname{dim} H$.

\section{REFERENCES}

[A] M. Auslander, Representation dimension of artin algebras, Queen Mary College, Mathematics Notes, University of London, 1971.

[AH] I. Assem, D. Happel, Generalized tilted algebras of type $\mathbb{A}_{n}$, Comm. Algebra 9 (1981), no. 20, 2101-2125. MR640613 (83a:16023a)

[APT] I. Assem, M. I. Platzeck, S. Trepode, On the representation dimension of tilted and laura algebras, J. Algebra 296 (2006), no. 2, 426-439. MR2201050 (2006k:16024)

[ARS] M. Auslander, I. Reiten, S. Smalø, Representation theory of Artin algebras, Cambridge University Press, 1995. MR 1314422 (96c:16015)

[AS1] M. Auslander, S. Smalø, Almost split sequences in subcategories, J. Algebra 69 (1981), no. 2, 426-454. MR617088 (82j:16048a)

[AS2] M. Auslander, S. Smalø, Preprojective modules over Artin algebras, J. Algebra 66 (1980), no. 1, 61-122. MR.591246 (83a:16039)

[CP] F. U. Coelho, M. I. Platzeck, On the representation dimension of some classes of algebras, J. Algebra 275 (2004), 615-628. MR2052629 (2005c:16019)

[EHIS] K. Erdmann, Th. Holm, O. Iyama, J. Schröer, Radical embeddings and representation dimension, Adv. Math. 185 (2004), no. 1, 159-177. MR2058783 (2005g:16019)

[GL1] W. Geigle, H. Lenzing, Perpendicular categories with applications to representations and sheaves, J. Algebra 144 (1991), no. 2, 273-343. MR.1140607(93b:16011)

[GL2] W. Geigle, H. Lenzing, A class of weighted projective curves arising in representation theory of finite-dimensional algebras, Singularities, representation of algebras, and vector bundles (Lambrecht, 1985), 265-297, Lecture Notes in Math., 1273, Springer, Berlin, 1987. MR 915180 (89b:14049)

[H1] D. Happel, Triangulated categories in the representation theory of finite-dimensional algebras, London Math. Soc. Lecture Notes Series, 119, Cambridge Univ. Press, Cambridge, 1988. MR 935124 (89e:16035)

[H2] D. Happel, A characterization of hereditary categories with tilting object, Invent. Math. 144 (2001), 381-398. MR1827736 (2002a:18014) 
[HRS] D. Happel, J. Rickard, A. Schofield, Piecewise hereditary algebras, Bull. London Math. Soc. 20 (1988), no. 1, 23-28. MR.916069 (89c:16039)

[HZ] D. Happel, D. Zacharia, Homological properties of piecewise hereditary algebras, J. of Algebra, to appear.

[I] O. Iyama, Finiteness of representation dimension, Proc. Amer. Math. Soc. 131 (2003), 1011-1014. MR.1948089 (2003k:16024)

[O] S. Oppermann, Representation dimension of quasitilted algebras, preprint.

[Ri] J. Rickard, Morita theory for derived categories, J. London Math. Soc. (2) 39 (1989), no. 3, 436-456. MR1002456 (91b:18012)

[R] C. M. Ringel, Tame algebras and integral quadratic forms, Lecture Notes in Mathematics, 1099, Springer-Verlag, Berlin-Heidelberg, 1984. MR774589 (87f:16027)

[S] U. Seidel, On tilting complexes and piecewise hereditary algebras, Dissertation Technische Universität Chemnitz, Chemnitz, 2003.

Departamento de Matemática - IMe, Universidade de São Paulo, CP 66281 São Paulo, BRAZIL

E-mail address: fucoelho@ime.usp.br

Fakultät für Mathematik, Technische Universität Chemnitz, D-09107 Chemnitz, GERMAnY

E-mail address: happel@mathematik.tu-chemnitz.de

Fakultät für Mathematik und Informatik, Fernuniversität Hagen, D-58084 Hagen, GERMANY

E-mail address: luise.unger@fernuni-hagen.de 